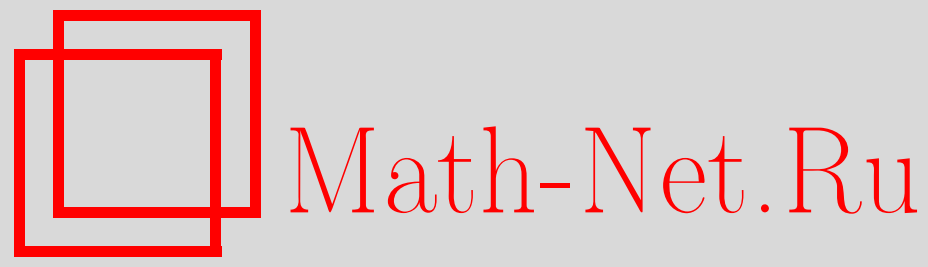

Э. Л. Пресман, Оценка константы в неравенстве Буркхольдера для супермартингалов и мартингалов, Теория вероятн. и ее примен., 2008, том 53, выпуск $1,172-178$

DOI: https://doi.org/10.4213/tvp2492

Использование Общероссийского математического портала Math-Net.Ru подразумевает, что вы прочитали и согласны с пользовательским соглашением

http://www . mathnet.ru/rus/agreement

Параметры загрузки:

IP : 52.6 .47 .48

26 апреля 2023 г., 17:36:59

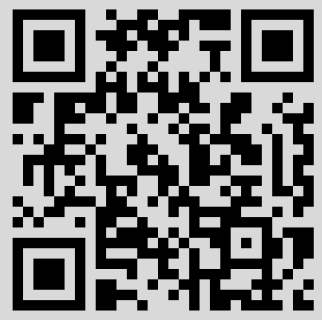


3. Artzner P., Delbaen F., Eber J.-M., Heath D. Coherent measures of risk. - Math. Finance, 1999, v. 9, № 3, p. 203-228.

4. Cherny A.S. Weighted V@R and its properties. - Finance Stoch., 2006, v. 10, № 2, p. $367-393$.

5. Cherny A.S., Madan D. B. Coherent measurement of factor risks. http://arxiv.org: math/0605062v1

6. Jung J. On linear estimates defined by a continuous weight function. - Ark. Mat., 1956 , v. 3, p. 199-209.

7. Lehmann E. L. Some concepts of dependence. - Ann. Math. Statist., 1966, v. 37, p. $1137-1153$.

8. Föllmer H., Schied A. Stochastic Finance. An Introduction in Discrete Time. Berlin: de Gruyter, 2004, 459 p.

9. Stigler S. M. Linear functions of order statistics with smooth weight functions. - Ann. Statist., 1974, v. 2, № 4, p. 676-693; corrections: ibid., 1979, v. 7, № 2, p. 466.

Поступила в редакцию

29.VI. 2007

(c) 2008 r.

ПРЕСМАН Э. Л.*

\section{ОЦЕНКА КОНСТАНТЫ В НЕРАВЕНСТВЕ БУРКХОЛЬДЕРА ДЛЯ СУПЕРМАРТИНГАЛОВ И МАРТИНГАЛОВ ${ }^{1)}$}

Доказывается моментное неравенство типа Буркхольдера для супермартингалов и мартингалов. Доказательство сводится к усовершенствованию соответствующих рассуждений С. В. Нагаева, который получил это неравенство с бо́льшим значением константы.

Ключевые слова и фразы: математическое ожидание, мартингал, супермартингал, неравенство Буркхольдера, моменты.

1. Формулировка и обсуждение результатов. Пусть последовательность случайных величин $S_{n}=\sum_{k=0}^{n} X_{k}, n \geqslant 0$, образует супермартингал, заданный на фильтрованном вероятностном пространстве $\left(\Omega, \mathscr{F},\left(\mathscr{F}_{k}\right)_{k \geqslant 0}, \mathbf{P}\right)$, где $X_{0}=0, \mathscr{F}_{0}=$ $\{\varnothing, \Omega\}$, так что $\mathbf{E}\left\{X_{k+1} \mid \mathscr{F}_{k}\right\} \leqslant 0, k \geqslant 0$. Положим

$$
B_{n}^{2}=\sum_{k=1}^{n} \mathbf{E}\left\{X_{k}^{2} \mid \mathscr{F}_{k-1}\right\}, \quad \bar{X}_{n}=\max _{0 \leqslant k \leqslant n} X_{k}, \quad \bar{S}_{n}=\max _{0 \leqslant k \leqslant n} S_{k} .
$$

Теорема 1. При $t \geqslant 1$ справедливо следующее неравенство:

$$
\frac{\left(\mathbf{E} \bar{S}_{n}^{t}\right)^{1 / t}}{\left(\mathbf{E} \bar{X}_{n}^{t}\right)^{1 / t}+\left(\mathbf{E} B_{n}^{t}\right)^{1 / t}} \leqslant c_{t}:=\inf _{\alpha>1} \varphi_{t}(\alpha)
$$

где

$$
\varphi_{t}(\alpha)=\alpha \frac{\exp \left(2 t^{-1}(\alpha(\ln \alpha-1)+1)\right)}{\left[\exp \left((t+1)^{-1}(\alpha(\ln \alpha-1)+1)\right)-1\right]^{(t+1) / t}} .
$$

Доказательство этой теоремы проводится в п. 2 усовершенствованием рассуждений из работы С.В. Нагаева [1], где, в частности, доказывалось соответствующее неравенство с другой функцией, которая, как показано ниже, заведомо больше $c_{t}$.

Пусть $S_{k}, k \geqslant 0,-$ мартингал, $\widehat{S}_{n}=\max _{1 \leqslant k \leqslant n}\left|S_{k}\right|, D_{t}=\mathbf{E}\left(\max _{1 \leqslant k \leqslant n}\left|X_{k}\right|^{t}\right)$.

Стандартным видоизменением доказательства теоремы 1 (см., например, [1]) получаем следующее утверждение.

* Центральный экономико-математический институт РАН, ул. Красикова, 47, 117418 Москва, Россия; e-mail: presman@cemi.ras.ru

1) Работа выполнена при поддержке Российского фонда фундаментальных исследований (гранты № 07-01-00541_а и 08-01-90252-Узб_а). 
Следствие 1. Если $S_{k}, k \geqslant 0,-$ мартингал, mо

$$
\left(\mathbf{E} \widehat{S}_{n}^{t}\right)^{1 / t} \leqslant c_{t}\left(D_{t}^{1 / t}+2^{1 / t}\left(\mathbf{E} B_{n}^{t}\right)^{1 / t}\right) .
$$

Это неравенство получил в 1973 г. Д.Л. Буркхольдер [2] без явного указания функции $c_{t}$. В 1990 г. П. Хитченко [3] показал, что при $t \geqslant 2$ справедливо неравенство

$$
\left(\mathbf{E} \widehat{S}_{n}^{t}\right)^{1 / t}<K \frac{t}{\ln t}\left(D_{t}^{1 / t}+\left(\mathbf{E} B_{n}^{t}\right)^{1 / t}\right)
$$

где $K$ - абсолютная постоянная. В 1997 г. Р. Ибрагимов и Ш. Шарахметов [4] показали, что если $X_{k}$ - независимые симметрично распределенные случайные величины, то

$$
\lim _{t \rightarrow \infty} \frac{\ln t}{t} \frac{\left(\mathbf{E} \widehat{S}_{n}^{t}\right)^{1 / t}}{D_{t}^{1 / t}+\left(\mathbf{E} B_{n}^{t}\right)^{1 / t}}=\frac{1}{e} .
$$

Подробный обзор моментных неравенств для мартингалов см. в [5].

С учетом соотношений (1) и (2) представляет интерес рассмотреть функцию

$$
K_{t}:=\frac{\ln t}{t} \inf _{\alpha>1} \varphi_{t}(\alpha)
$$

Теорема 2. (а) При каждом $t>0$ существует такое число $a_{t}>1$, что функиия $\varphi_{t}(\alpha)$ убывает при $1<\alpha<a_{t}$ и возрастает при $\alpha>a_{t} ;$ бункиия $a_{t}$ является единственным решением уравнения

$$
-a+1+t x_{t}(a)-(1+t)\left(\ln \left(1+2 x_{t}(a)\right)-\ln \left(1+x_{t}(a)\right)\right)=0,
$$

где $x_{t}(a)=(a \ln a) / t$, при этом

$$
\frac{a_{t}(\ln t)^{2}}{t}=\frac{2}{3}\left(1+4 \frac{\ln \ln t}{\ln t}+\frac{28 / 27+\ln (9 / 4)}{\ln t}+O\left(\frac{(\ln \ln t)^{2}}{(\ln t)^{2}}\right)\right) \quad \text { npu } \quad t \rightarrow \infty .
$$

(б) Функция $K_{t}=(\ln t) \varphi_{t}\left(a_{t}\right) / t$ убьввает при $t \geqslant 2$ от $K_{2} \approx 9.46$ до 1 .

Доказательство этой теоремы проводится в п. 3 .

Отметим, что соответствуюшая $K_{t}$ функция, фигурирующая в $[1]$, которую мы обозначаем $\widetilde{K}_{t}$, убывает на интервале $2<t<20.1$, возрастает на интервале $20.1<$ $t<49.94$ и убывает при $t>49.94$ до значения 1.77 .

В приводимой ниже таблице сравниваются значения упомянутых функций.

\begin{tabular}{|c|c|c|c|c|c|c|c|c|c|c|}
\hline$t$ & 2 & 3 & 8 & 20 & 50 & 200 & 1000 & $10^{5}$ & $10^{18}$ & $\infty$ \\
\hline$K_{t}$ & 9.46 & 8.86 & 6.70 & 5.33 & 4.43 & 3.56 & 2.93 & 2.10 & 1.29 & 1 \\
\hline$\widetilde{K}_{t}$ & 28.74 & 23.09 & 14.96 & 12.76 & 13.47 & 5.95 & 4.09 & 2.78 & 1.98 & 1.77 \\
\hline
\end{tabular}

2. Доказательство теоремы 1. Для доказательства нам понадобятся два предложения, которые приведены в [1] в несколько иной формулировке.

Пусть $Q(x)=\mathbf{P}\left\{\bar{X}_{n}>x\right\}+\mathbf{P}\left\{B_{n}>x\right\}$.

Предложение 1. При всех $x>0, y>0, \alpha>1$ выполняется неравенство

$$
\mathbf{P}\left\{\bar{S}_{n} \geqslant x+\alpha y\right\}<\mathbf{P}\left\{\bar{S}_{n} \geqslant x\right\} e^{-(\alpha(\ln \alpha-1)+1)}+Q(y) .
$$

Именно это предложение доказано в [1], однако в формулировке там вместо множителя $e^{-(\alpha(\ln \alpha-1)+1)}$, который всегда меньше единицы, фигурирует $e^{-\alpha(\ln \alpha-1)}$.

Предложение 2. Пусть неотричательная функиия $A(x)$ не возрастает при $x>0$, имеет конечный предел при $x \downarrow 0 u$

$$
A(t, x)=\frac{1}{x^{t}} \int_{0}^{x} A(u) u^{t-1} d u=\int_{0}^{1} A(x u) u^{t-1} d u .
$$


Если Функция $B(x)$ имеет конечный верхний предел при $x \downarrow 0$ и существуют такие $\alpha>0$ и $0<p<1$, что при всех $x, y>0$ выполняется неравенство

$$
B(x+\alpha y) \leqslant p B(x)+A(y)
$$

то для любого $x>0$ и любьх таких $\varepsilon$ и $\widetilde{p}$, ито $p \leqslant \widetilde{p}<1 u 1+\alpha \varepsilon<1 / \widetilde{p}$, справедливо

$$
B(x) \leqslant \frac{1}{\alpha \varepsilon \widetilde{p}^{2}} A\left(-\frac{\ln \tilde{p}}{\ln (1+\alpha \varepsilon)}, \varepsilon x\right) .
$$

Д о к а з а т е л ь с т в о. Неравенство (7) доказано в [1] для $\widetilde{p}=p=e^{-\alpha(\ln \alpha-1)}$ и для конкретных $A(x)=Q(x)$ и $B(x)=\mathbf{P}\left\{\bar{S}_{n} \geqslant x\right\}$, для которых, согласно предложению 1 , неравенство (6) выполняется при любом $\alpha>1$ и $p=e^{-\alpha(\ln \alpha-1)}$, при этом вместо $\alpha \varepsilon \widetilde{p}^{2}$ там фигурирует $\alpha \varepsilon \widetilde{p}^{3}$. Приводимое ниже доказательство лишь незначительно отличается от доказательства из [1].

Очевидно, что достаточно рассмотреть случай $p=\widetilde{p}$. Пусть $\varepsilon$ и $x$ фиксированы. Фиксируем целое число $m>1$ и рассмотрим последовательность

$$
y_{k}=(1+\alpha \varepsilon)^{k-m} x, \quad k \geqslant 0,
$$

для которой $y_{m}=x$. Очевидно, что

$$
y_{k}-y_{k-1}=\alpha \varepsilon y_{k-1} .
$$

Подставляя в правую часть (6) в качестве аргумента первого слагаемого $y_{k-1}$, а в качестве аргумента второго слагаемого $\varepsilon y_{k-1}$ и используя (9), получаем

$$
B\left(y_{k}\right) \leqslant B\left(y_{k-1}\right) p+A\left(\varepsilon y_{k-1}\right) .
$$

Отсюда следует, что

$$
B(x)=B\left(y_{m}\right) \leqslant \sum_{1}^{m-1} A\left(\varepsilon y_{k}\right) p^{m-k-1}+B\left(y_{1}\right) p^{m-1} .
$$

Положим

$$
s:=-\frac{\ln p}{\ln (1+\alpha \varepsilon)}>1
$$

Используя сначала (9), затем (8) и, наконец, равенство $1 / p=(1+\alpha \varepsilon)^{s}$, которое следует из (11), получим

$$
\frac{1}{x^{s}}\left(y_{k-1}\right)^{s-1}\left(y_{k}-y_{k-1}\right)=\alpha \varepsilon\left(\frac{y_{k-1}}{x}\right)^{s}=\alpha \varepsilon\left((1+\alpha \varepsilon)^{s}\right)^{k-1-m}=\alpha \varepsilon p^{m-k+1} .
$$

Из этого равенства и того, что функции $A(x)$ и $x^{s-1}$ не возрастают, следует, что

$$
A\left(\varepsilon y_{k}\right) p^{m-k-1}=\frac{1}{\alpha \varepsilon p^{2}} \frac{1}{x^{s}} A\left(\varepsilon y_{k}\right)\left(y_{k-1}\right)^{s-1}\left(y_{k}-y_{k-1}\right) \leqslant \frac{1}{\alpha \varepsilon p^{2}} \frac{1}{x^{s}} \int_{y_{k-1}}^{y_{k}} A(\varepsilon u) u^{s-1} d u .
$$

Отсюда, а также из (10) и неотрицательности функции $A(x)$ следует

$$
B(x) \leqslant \frac{1}{\alpha \varepsilon p^{2}} \frac{1}{x^{s}} \int_{0}^{x} A(\varepsilon u) u^{s-1} d u+B\left(y_{1}\right) p^{m-1} .
$$

Используя ограниченность величины $\lim \sup _{x \downarrow 0} B(x)$, в соотношении (13) можно перейти к пределу при $m \rightarrow \infty$, что завершает доказательство предложения 2 .

Отметим, что в работе [1] рассматривалась последовательность $y_{k}=\beta(1+\alpha \varepsilon)^{k}$ для такого $\beta$, что $y_{m} \leqslant x<y_{m+1}$, а в равенстве (12) вместо $x$ фигурировало $y_{m+1}$. Именно за счет этого там получалось $p^{3}$ вместо $p^{2}$. Кроме того, в работе [1] использовалась монотонность функции $B(x)$, которую мы не требуем.

Согласно второму равенству в $(5)$, функция $A(t, x)$ не возрастает по $t$. Поэтому если $1+\alpha \varepsilon \leqslant p^{-1 /(t+1)}$, то можно взять $\widetilde{p}=e^{-(t+1) \ln (1+\alpha \varepsilon)}$, а значит,

$$
B(x) \leqslant \frac{(1+\alpha \varepsilon)^{2(t+1)}}{\alpha \varepsilon} \frac{1}{(\varepsilon x)^{t+1}} \int_{0}^{\varepsilon x} A(u) u^{t} d u
$$

при $1+\alpha \varepsilon \leqslant p^{-1 /(t+1)}$. Домножая (14) на $x^{t-1}$, интегрируя по $x$ от 0 до $\infty$ и меняя в правой части порядок интегрирования, получаем следующий результат. 
Следствие 2. Если $t>0$, а $A(x)$ и $B(x)$ удовлетворяют условиям предложения 2 , то для любого такого $\varepsilon$, ито $\varepsilon>0,1+\alpha \varepsilon \leqslant p^{-1 /(t+1)}$, выполняется неравенство

$$
\int_{0}^{\infty} B(x) x^{t-1} d x \leqslant \frac{(1+\alpha \varepsilon)^{2(t+1)}}{(\alpha \varepsilon)^{t+1}} \alpha^{t} \int_{0}^{\infty} A(x) x^{t-1} d x .
$$

Рассмотрим теперь случай $B(x)=\mathbf{P}\left\{\bar{S}_{n} \geqslant x\right\}, A(x)=Q(x)$, когда неравенство (6) выполняется для любого $\alpha>1$ при $p=p(\alpha)=e^{-(\alpha(\ln \alpha-1)+1)}$. В этом случае при фиксированном $t$ коэффициент при интеграле в правой части (15) можно рассматривать как функцию двух переменных $x=\alpha \varepsilon$ и $\alpha$, которая при фиксированном $x$ достигает минимума при минимально возможном значении $\alpha$, а значит, минимум по области достигается на ее границе, т.е. на кривой $1+\alpha \varepsilon=(p(\alpha))^{-1 /(t+1)}$. Домножая неравенство (15) на $t$, возводя обе части в степень $1 / t$ и используя элементарное неравенство, связывающее момент суммы и сумму моментов, получаем утверждение теоремы.

Заметим, что в работе [1] при фиксированном $t$ рассматривались не оптимальные, а некоторые конкретные значения $\alpha_{t}$ и $\varepsilon_{t}$. Поэтому наша константа заведомо не хуже константы, полученной в работе [1].

3. Доказательство теоремы 2. Функцию $\varphi_{t}(\alpha)$ можно представить в виде

$$
\varphi_{t}(a)=a f^{(t+1) / t}\left(u_{t}(a)\right), \quad \text { где } \quad f(u)=\frac{u^{2}}{u-1}, \quad u_{t}(a)=\exp \left(\frac{a(\ln a-1)+1}{t+1}\right)
$$

Используя это представление, переходя к логарифмам и учитывая равенства

получаем

$$
\frac{d}{d u} \ln f(u)=\frac{u-2}{(u-1) u} \quad \text { и } \quad \frac{\partial u_{t}(a)}{\partial a}=\frac{\ln a}{t+1} u_{t}(a),
$$

$$
\frac{1}{\varphi_{t}(a)} \frac{\partial}{\partial a} \varphi_{t}(a)=\frac{1}{a}\left(1+\frac{a \ln a}{t} \frac{u_{t}(a)-2}{u_{t}(a)-1}\right)=\frac{1}{a} \frac{1+(2 a \ln a) / t}{u_{t}(a)-1}\left[\exp \left(\frac{b_{t}(a)}{t+1}\right)-1\right]
$$
где

$$
\begin{aligned}
b_{t}(a) & =b_{t}\left(a, x_{t}(a)\right), \quad x_{t}(a)=\frac{a \ln a}{t}, \\
b_{t}(a, x) & =-a+1+t x-(t+1)(\ln (1+2 x)-\ln (1+x)) .
\end{aligned}
$$

Поскольку $\partial x_{t}(a) / \partial a=(1+\ln a) / t$, то

$$
\begin{aligned}
\frac{\partial}{\partial a} b_{t}(a) & =-1+\left(1-\frac{t+1}{t} \frac{1}{1+2 x_{t}(a)} \frac{1}{1+x_{t}(a)}\right)(1+\ln a) \\
& =\ln a\left[1-\frac{1+\ln a}{\ln a} \frac{t+1}{t} \frac{1}{1+2 x_{t}(a)} \frac{1}{1+x_{t}(a)}\right] .
\end{aligned}
$$

При возрастании $a$ от 1 до $+\infty$ выражение в квадратных скобках растет от $-\infty$ до 1 , а поскольку $b_{t}(1)=0$, то функция $b_{t}(a)$ сначала убывает и отрицательна, а потом возрастает до $+\infty$. А значит, сушествует такое $a_{t}$, что

$$
b_{t}\left(a_{t}\right) \equiv 0, \quad b_{t}(a)<0 \quad \text { при } \quad 1<a<a_{t}, \quad b_{t}(a)>0 \quad \text { при } \quad a>a_{t} .
$$

Эта точка является точкой минимума функции $\varphi_{t}(a)$. Тем самым доказаны существование $a_{t}$ и тот факт, что $a_{t}$ является единственным решением уравнения (3).

Пусть

$$
\tilde{a}_{t}=\frac{2 t}{3(\ln t)^{2}}\left(1+4 \frac{\ln \ln t}{\ln t}+\frac{c}{\ln t}\right)
$$

где $c$ ограничено. Тогда

$$
\begin{aligned}
x_{t}\left(\tilde{a}_{t}\right) & =\frac{a_{t} \ln a_{t}}{t}=\frac{2}{3(\ln t)^{2}}\left(1+4 \frac{\ln \ln t}{\ln t}+\frac{c}{\ln t}\right)\left(\ln t+\ln \frac{2}{3}-2 \ln \ln t+O\left(\frac{\ln \ln t}{\ln t}\right)\right) \\
& =\frac{2}{3 \ln t}\left(1+2 \frac{\ln \ln t}{\ln t}+\frac{c+\ln (2 / 3)}{\ln t}+O\left(\frac{\ln \ln t}{(\ln t)^{2}}\right)\right), \\
x_{t}^{2}\left(\widetilde{a}_{t}\right) & =\frac{4}{9(\ln t)^{2}}\left(1+4 \frac{\ln \ln t}{\ln t}+2 \frac{c+\ln (2 / 3)}{\ln t}+O\left(\frac{(\ln \ln t)^{2}}{(\ln t)^{2}}\right)\right) .
\end{aligned}
$$


Используя формулу Тейлора до членов третьего порядка, получаем из (18) и (19), что

$$
b_{t}(a)=-a+1+\frac{3}{2}(t+1) x_{t}^{2}(a)\left(1-\frac{14}{9} x_{t}(a)+O\left(x_{t}^{2}(a)\right)\right)-x_{t}(a) .
$$

Подставляя (21)-(23) в (24), получаем, что $b_{t}\left(\widetilde{a}_{t}\right) \cdot 3(\ln t)^{3} /(2 t)=c-\frac{28}{27}-2 \ln (3 / 2)+$ $O\left((\ln \ln t)^{2} / \ln t\right)$. Отсюда с учетом (21) и (20) получаем (4), что завершает доказательство п. (a) теоремы 2.

Перейдем к доказательству п. (б). Заметим сначала, что из представления (16) после перехода к логарифмам с учетом того, что $d \ln f(u) / d u=(u-2) /((u-1) u)$ и $\partial u_{t}(a) / \partial t=-u_{t}(a)\left(a_{t}\left(\ln a_{t}-1\right)+1\right) /(t+1)^{2}$, получаем

$$
\begin{aligned}
\left.\frac{1}{\varphi_{t}\left(a_{t}\right)} \frac{\partial \varphi_{t}(a)}{\partial t}\right|_{a=a_{t}} & =\left.\left[\frac{\partial}{\partial t}\left(\frac{t+1}{t} \ln f\left(u_{t}(a)\right)\right)\right]\right|_{a=a_{t}} \\
& =\left[-\frac{1}{t^{2}} \ln \frac{u_{t}^{2}\left(a_{t}\right)}{u_{t}\left(a_{t}\right)-1}-\frac{t+1}{t} \frac{u_{t}\left(a_{t}\right)-2}{u_{t}\left(a_{t}\right)-1} \frac{a_{t}\left(\ln a_{t}-1\right)+1}{(t+1)^{2}}\right] .
\end{aligned}
$$

Положим $u_{t}=u_{t}\left(a_{t}\right), x_{t}=\left(a_{t} \ln a_{t}\right) / t$. Из первого соотношения в (20) и из (17) следует, что $\left.\left[\partial \varphi_{t}(a) / \partial a\right]\right|_{a=a_{t}}=0$. Отсюда и из первого соотношения в (17) получаем $\frac{u_{t}-2}{u_{t}-1}=-\frac{1}{x_{t}}, \quad u_{t}=\frac{1+2 x_{t}}{1+x_{t}}, \quad u_{t}-1=\frac{x_{t}}{1+x_{t}}, \quad \frac{a_{t}\left(\ln a_{t}-1\right)+1}{t+1}=\ln \frac{1+2 x_{t}}{1+x_{t}}$, при этом последнее равенство следует из (18), (19) и первого равенства в (20).

Подставляя первые три равенства из (26) в (25) и учитывая, что $a_{t} \ln a_{t}=t x_{t}$, полтучаем

$$
\left.\frac{\partial \varphi_{t}(a)}{\partial t}\right|_{a=a_{t}}=\frac{\varphi_{t}\left(a_{t}\right)}{t}\left[\frac{1}{x_{t}} \frac{t x_{t}-a_{t}+1}{t+1}-\frac{1}{t} \ln \frac{\left(1+2 x_{t}\right)^{2}}{x_{t}\left(1+x_{t}\right)}\right] .
$$

Дифференцирование равенства $K_{t}=\left(\varphi_{t}\left(a_{t}\right) \ln t\right) / t$ дает

$$
\frac{d K_{t}}{d t}=\frac{1-\ln t}{t^{2}} \varphi_{t}\left(a_{t}\right)+\frac{\ln t}{t}\left(\left.\frac{\partial \varphi_{t}(a)}{\partial t}\right|_{a=a_{t}}+\left.\frac{\partial \varphi_{t}(a)}{\partial a}\right|_{a=a_{t}} \frac{d a_{t}}{d t}\right) .
$$

С учетом $(27)$ и того, что $\left.\left[\partial \varphi_{t}(a) / \partial a\right]\right|_{a=a_{t}}=0$, получаем, что $d K_{t} / d t=\varphi_{t}\left(a_{t}\right)(1-$ $\left.\Psi_{t}\right) / t^{2}$, где

$$
\frac{\Psi_{t}}{\ln t}=1-\frac{1}{x_{t}} \frac{t x_{t}-a_{t}+1}{t+1}+\frac{1}{t} \ln \frac{\left(1+2 x_{t}\right)^{2}}{x_{t}\left(1+x_{t}\right)}=\frac{a_{t}-1}{t x_{t}}+\frac{t x_{t}-a_{t}+1}{t(t+1) x_{t}}+\frac{1}{t} \ln \frac{\left(1+2 x_{t}\right)^{2}}{x_{t}\left(1+x_{t}\right)} .
$$

В последнем равенстве мы воспользовались тем, что $(t+1)^{-1}=t^{-1}-(t(t+1))^{-1}$.

Таким образом, для доказательства убывания функции $K_{t}$ при $t>2$ достаточно показать, что $\Psi_{t}>1$ при $t>2$. Положим

$$
\begin{aligned}
\Psi_{1}(y) & =\frac{y \ln y}{y-1} \quad \text { при } \quad y>1, \\
\Psi_{2}(y) & =\frac{1}{y} \ln \frac{1+2 y}{1+y}, \quad \Psi_{3}(y)=\ln \frac{(1+2 y)^{2}}{y(1+y)} \quad \text { при } \quad y>0, \\
\Psi_{t}(a, x) & =\frac{\ln t}{\Psi_{1}(a)}+\frac{\ln t}{t}\left(\Psi_{2}(x)+\Psi_{3}(x)\right) .
\end{aligned}
$$

Подставляя в правую часть (29) последнее равенство из (26), с учетом равенства $a_{t} \ln a_{t}=t x_{t}$ получаем, что

$$
\Psi_{t}=\Psi_{t}\left(a_{t}, x_{t}\right)
$$

Отметим, что функции $\Psi_{i}(y), i=1,2,3$, положительны, функция $\Psi_{1}(y)$ возрастает, а функции $\Psi_{2}(y)$ и $\Psi_{3}(y)$ убывают. Отсюда и из соотношений (31) и (31) вытекает, что если нам удастся построить такую функцию $\widehat{a}_{t}$, что

$$
\widehat{a}_{t}>a_{t}, \quad \Psi_{t}\left(\widehat{a}_{t}, x_{t}\left(\widehat{a}_{t}\right)\right)>1,
$$

то убывание функции $K_{t}$ при $t>2$ будет доказано. 
Мы утверждаем, что при $t \geqslant 2.1$ функция $\bar{a}_{t}=(t+5) / \ln (t+5)$ удовлетворяет (32). Покажем сначала, что для $\widehat{a}_{t}=\bar{a}_{t}$ первое неравенство в $(32)$ выполняется при $t \geqslant 2$. Из (18)-(20) следует, что для этого достаточно доказать, что $b_{t}\left(\bar{a}_{t}, x_{t}\left(\bar{a}_{t}\right)\right)>0$. Заметим сначала, что $\bar{x}_{t}:=x_{t}((t+5) / \ln (t+5))=(1+5 / t)(1-(\ln \ln (t+5)) / \ln (t+5))$.

Из того, что функция $(\ln x) / x$ при $x=e$ достигает максимума, равного $1 / e$, следует, что $\bar{x}_{t} \geqslant 1-(1 / e)>0.632$. В рассматриваемом случае

$$
\bar{b}_{t}=b_{t}\left(\bar{a}_{t}, \bar{x}_{t}\right)=-\frac{t+5}{\ln (t+5)}+1-\ln \frac{1+2 \bar{x}_{t}}{1+\bar{x}_{t}}+t\left(\bar{x}_{t}-\ln \frac{1+2 \bar{x}_{t}}{1+\bar{x}_{t}}\right) .
$$

Из полученной оценки для $\bar{x}_{t}$ и возрастания функций $x-\ln ((1+2 x) /(1+x))$ и $\ln ((1+$ $2 x) /(1+x))$ следует, что $\bar{b}_{t} \geqslant-(t+5) / \ln (t+5)+1-\ln 2+t[0.632-\ln ((1+1.264) /(1+$ $0.632))]$. Функция в правой части последнего неравенства положительна при $t \geqslant 34$.

Нетрудно проверить, что функция $\bar{x}_{t}$ убывает на интервале $[2,34]$. Отсюда, из (33) и возрастания функций $x / \ln x, x-\ln ((1+2 x) /(1+x))$ и $\ln ((1+2 x) /(1+x))$ получаем, что если $k<t \leqslant k+1$, то

$$
\bar{b}_{t}>-\frac{k+1+5}{\ln (k+1+5)}+1-\ln \frac{1+2 \bar{x}_{k+1}}{1+\bar{x}_{k+1}}+k\left(\bar{x}_{k}-\ln \frac{1+2 \bar{x}_{k}}{1+\bar{x}_{k}}\right)=: c_{k} .
$$

Непосредственное вычисление (например, с использованием EXCEL) показывает, что $c_{k}>0$ при $2 \leqslant k<34$. Это завершает доказательство первого неравенства в (32).

Для доказательства второго неравенства в (32) покажем, что $(\ln t) / \Psi_{1}\left(\bar{a}_{t}\right)>1$ при $t \geqslant 11$. Из положительности функций $\Psi_{2}(x)$ и $\Psi_{3}(x)$ и из $(31)$ следует, что отсюда будет вытекать выполнение второго неравенства в (32) при $t \geqslant 11$. Неравенство $(\ln t) / \Psi_{1}\left(\bar{a}_{t}\right)>1$ эквивалентно неравенству $\ln \ln (t+5)-\ln (1+5 / t)-(\ln t \ln (t+5)) /$ $(t+5)>0$, которое, очевидно, вытекает из неравенства $\ln \ln (t+5)-\ln (1+5 / t)-(\ln (t+$ $5))^{2} /(t+5)>0$. Функция, стоящая в левой части последнепо неравенства, возрастает и при $t=11$ принимает значение 0.069. Тем самым доказано выполнение второго неравенства в (32) при $t \geqslant 11$. Доказательство для $2.1 \leqslant t<11$ проводится аналогично доказательству того, что $\bar{b}_{t}>0$ на отрезке $[2,34]$, при этом надо учитывать функции $\Psi_{2}(x)$ и $\Psi_{3}(x)$.

Заметим теперь, что непосредственный подсчет показывает, что $\Psi_{2} \approx 1.002$, $\Psi_{2.1} \approx 1.004$, в то время как $\Psi_{2}\left(\bar{a}_{2}, x_{2}\left(\bar{a}_{2}\right)\right) \approx 0.962, \Psi_{2.1}\left(\bar{a}_{2.1}, x_{2.1}\left(\bar{a}_{2.1}\right)\right) \approx 1.003$. Таким образом при $2<t<2.1$ в качестве $\widehat{a}_{t}$ нужно использовать функцию, отличную от $\bar{a}_{t}$. Можно использовать линейное приближение. Доказательство носит аналогичный характер, и мы его опускаем. Тем самым доказано, что $K_{t}$ убывает при $t>2$.

Значение $K_{2}$ получается из того, что $a_{2} \approx 3.18$. Последний факт следует из того, что это значение обращает в нуль функцию $b_{2}\left(a, x_{2}(a)\right)$ из (18).

Осталось показать, что $\lim _{t \rightarrow \infty} K_{t}=1$. Из определения функции $K_{t}$, представления (16) и соотношений (26) получаем, что

$$
K_{t}=\frac{\ln t}{t} a_{t}\left(\frac{\left(1+2 x_{t}\right)^{2}}{x_{t}\left(1+x_{t}\right)}\right)^{(t+1) / t} .
$$

Подстановка в это выражение главных частей из (21) и (8) завершает доказательство теоремы 2.

Автор выражает благодарность С. В. Нагаеву за полезные обсуждения.

\section{СПИСОК ЛИТЕРАТУРЫ}

1. Нагаев С. В. О вероятностных и моментных неравенствах для супермартингалов и мартингалов. - Теория вероятн. и ее примен., 2006, т. 51, в. 2, с. 391-400.

2. Burkholder D. L. Distribution function inequalities for martingales. - Ann. Probab., 1973 , v. 1, p. 19-42.

3. Hitczenko P. Best constants in martingale version of Rosenthal's inequality. - Ann. Probab., 1990, v. 18, № 4, p. 1656-1668. 
4. Ибрагимов P., Шарахметов Ш. Точная константа в неравенстве Розенталя для случайных величин с нулевым средним. - Теория вероятн. и ее примен., 2001, т. 46 , в. 1 , с. $134-138$.

5. Пешкир Г., Ширяев А.Н. Неравенства Хинчина и мартингальное расширение сферы их действия. - Успехи матем. наук, 1995, т. 50, № 5, с. 3-62.

Поступила в редакцию 15.VIII.2006

(C) 2008 г

ПУГАЧЕВ О.В.*

\section{ЕМКОСТИ И ПОВЕРХНОСТНЫЕ МЕРЫ В ЛОКАЛЬНО ВЫПУКЛЫХ ПРОСТРАНСТВАХ ${ }^{1)}$}

Доказывается плотность емкостей, порожденных соболевскими классами любого порядка в широком классе локально выпуклых пространств. Эти емкости применяются при построении поверхностных мер на множествах уровня соболевских функций и локальных соболевских функций.

Ключевые слова и фразы: дифференцируемая мера, соболевские классы в локально выпуклых пространствах, плотность емкости, поверхностная мера, формула Остроградского-Гаусса, локальные соболевские функции.

1. Введение. Поверхностные меры и емкости в бесконечномерных пространствах представляют интерес как для самой аналитической и геометрической теории меры, так и для ее приложений в нелинейном анализе, в теории случайных процессов, в теории линейных дифференциальных уравнений относительно функций и мер.

Первый подход к построению поверхностных мер в бесконечномерных пространствах берет начало в книге А. В. Скорохода [1], затем он был существенно развит в работах А. В. Угланова [2]-[4]. Он основан на построении локальных поверхностных мер в достаточно малых окрестностях точек поверхности.

Совершенно другой подход, реализованный Э. Эро и П. Маллявэном в работе [5] для винеровской меры и В.И. Богачевым [6]-[8] для дифференцируемых мер, в дальнейшем был развит автором. В этом методе мера строится сразу на всей поверхности, а условия гладкости поверхности связаны не с геометрией объемлюшего пространства, а с геометрией подпространства Камерона-Мартина, причем функция, задаюшая поверхность, принадлежит соболевскому классу, она даже не обязана быть непрерывной (таковы типичные функции, появляющиеся в теории случайных процессов).

В приложениях иногда встречаются функции, не являющиеся соболевскими глобально, но обладающие подобными свойствами локально. Цель данной работы обобщить результаты, полученные в [9], [10], для локальных соболевских функций и задаваемых ими поверхностей.

Новизна данной работы по сравнению с [9]-[11] также и в том, что результаты получены для более широкого класса локально выпуклых пространств.

2. Соболевские классы. Пусть $X-$ локально выпуклое пространство; пусть сепарабельное гильбертово пространство $H$ непрерывно вложено в $X$. Обозначим через $\langle\cdot ; \cdot\rangle$ скалярное произведение в $H$, через $|\cdot|$ - норму в $H$. Тогда формула

$$
j_{H}: X^{*} \rightarrow H^{*}=H, \quad\left\langle j_{H}(l) ; h\right\rangle=l(h) \quad \forall h \in H,
$$

* Московский государственный технический университет им. Н.Э. Баумана, 2-я Бауманская ул., 5, 105005 Москва, Россия; e-mail: opugachev@yandex.ru

1) Работа выполнена при поддержке грантов РФФИ 07-01-00536 и DFG 436 RUS 113/343/0(R). 